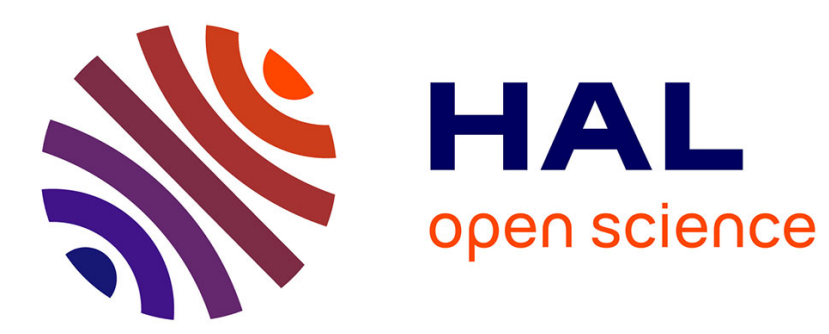

\title{
Observation d'un microcéphale vivant et de la cause probable de sa monstruosité
}

\author{
M.L. Manouvrier
}

\section{To cite this version:}

M.L. Manouvrier. Observation d'un microcéphale vivant et de la cause probable de sa monstruosité. Bulletins de la Société d'Anthropologie de Paris, 1895, pp.228-231. halshs-00848633

\section{HAL Id: halshs-00848633 \\ https://shs.hal.science/halshs-00848633}

Submitted on 26 Jul 2013

HAL is a multi-disciplinary open access archive for the deposit and dissemination of scientific research documents, whether they are published or not. The documents may come from teaching and research institutions in France or abroad, or from public or private research centers.
L'archive ouverte pluridisciplinaire HAL, est destinée au dépôt et à la diffusion de documents scientifiques de niveau recherche, publiés ou non, émanant des établissements d'enseignement et de recherche français ou étrangers, des laboratoires publics ou privés. 


\section{DE}

PALEONTOLOGIE

HUMAINE PARIS

(Extrail des Bullelins de la Soctété d'Antlıropologie de Paris).

\section{Observation d'nn microcéphale vivant et de la cause probable de sa monstruosité.}

Par M. L. Manouviter.

Il s'agit d'un enfant du sexe masculin (...Mey...), agé de 7 ans. Il est né à Paris de parents très bien conformés, d'origine alsacienne, qui habitent actuellement Aubervilliers. C'est sa mère qui est venue me le présenter et que j'ai pu interroger. Cette pauvre femme, très courageuse, gagne péniblement son pain et celui de sa famille en lavant du linge quand elle le peut, son mari étant en ce moment malade. Celui-ci exerce la profession d'ouvrier émailleur. Il est très rangé et n'est pas alcoolique. Pas de dégénérés ni dans sa famille ni dans celle de sa femme, d'après les renseignements fournis par cette dernière.

Ils ont eu quatre enfants dont le microcéphale est le second. Les autres étaient tous de très beaux enfants. Le troisième est mort à l'àge de deux mois avec des convulsions. Le premier est une jolie et intelligente petite fille, agée de neuf ans que je présente en mème temps que son frère microcéphale. Elle porte fièrement la croix d'honneur qu'elle vient de gagner à l'école communale. Le dernier né, dit la mère, est encore plus vigoureux. En somme, la famille parait ètre exempte de toute tare pathologique; c'est une famille que l'on peut dire physiquement florissante.

L'enfant microcéphale présente le facies caractéristique de cette monstruosité, facies qui résulte des deux caractères

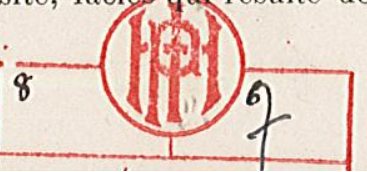


que j’ai indiqués ${ }^{1}$ comme différenciant la microcéphalie vraie de l'exiguité cérébrale en rapport avec l'exiguité de la taille, à savoir la petitesse, non seulement absolue, mais encore relative du cràne, par rapport à la taille et par rapport à la face.

La taille, que je n'ai pu mesurer à cause des mouvements du sujet, est ì peu près celle d'un enfant de 2 ans. Voici les principales mesures de la tête :

Diamètre antéro-postérieur maximum. 130 millimètres.

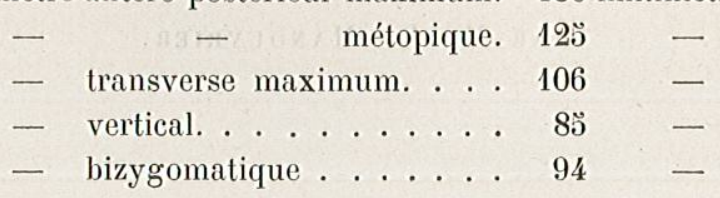

L'indide céphalique $=81.5$.

Comme l'indique la différence entre les deux diamètres antéro-postérieurs, le front est fuyant, caractère également régulier de la microcéphalie.

Au dire de la mère, le front était plus aplati lors de la naissance; il s'est bombé un peu à la partie supérieure.

Les globes oculaires sont un peu saillants; la mère affirme qu'il ont toujours été ainsi. Elle affirme également que la tète a beaucoup grossi depuis la naissance. Elle a remarqué, à cette époque, que la fontanelle bregmatique n'existait pas. En palpant le cràne, je trouve une saillie assez forte de la région du lambda.

Les quatre dents canines sont remarquablement pointues et dépassent sensiblement les dents yoisines. Ce caractère pithécoïde est assez accusé pour n'avoir pas échappé à l'observation de la mère.

Je n'ai pu le constater sur aucun des crànes microcéphales conservés au musée Broca; il est vrai que la plupart de ces crànes ont perdu leurs canines.

1 Article Microcéphalie du Dict. des Sciences Anthrop. Paris, Doin, éditeur.
La langue paraîtètre volumineuse; elle fait continuellement saillie derrière les lèvres entr'ouvertes.

La forme et les dimensions des oreilles sont normales.

L'enfant a eu plusieurs fois, dès l'àge de 2 ou 3 mois, des convulsions. Actuellement, sa santé est très bonne.

Sa main gauche présente comme particularité curieuse un seul pli palmaire transversal situé à peu près au milieu de la paume. C'est un caractère pithécoïde qui n'est peut-ètre pas extrèmement rare, car je l'ai rencontré jusqu'à présent, sans le chercher, sur deux hommes adultes et d'intelligence normale qui ont été présentés par moi à la Société d'Anthropologie.

La main droite ne présente pas le caractère ci-dessus : un second pli palmaire jrejoint le pli médian.

Ayant'été obligé, par suite d'un petit accident, de différer l'examen des organes génitaux, je n'ai point retrouvé l'occasion de faire cet examen.

L'enfant est complètement idiot et gateux. Il manifeste une bonne humeur remarquable, gràce aux soins attentifs de sa mère qui s'occupe de lui avec autant de sollicitude que s'il s'agissait d'un nourrisson normal et éducable. Il regarde les objets, mais sans attention et saisit machinalement ceux qu'on lui met dans la main. Parfois, il semble sourire à sa mère et semble reconnaitre aussi sa sœur. Il ne prononce aucune parole; il émet seulement des grognements un peu variés suivant qu'il souffre ou qu'il est satisfait.

Lorsqu'on le pose à terre, il se traîne assez vigoureusement, à la façon des enfants qui ne savent pas encore marcher. Il s'appuie, pour cela, sur la face palmaire des mains et jamais sur la face dorsale. Cet exercice, longtemps et fréquemment répété, a entrainé une torsion notable des avantbras et des jambes.

J'ai longuement interrogé la mère dans le but de saisir quelque cause à laquelle on puisse attribuer l'arrèt de développement évidemment intra-utérin de son enfant. Cette femme a simplement noté, comme événement insolite pendant 
sa grossesse anormale, qu'elle a eu un jour peur de chiens qui se battaient.

Je serais plus disposé à attacher quelque importance, soit au surmenage qu'elle a subi durant cette grossesse, soit aux pressions ou aux heurts subis par son abdomen, et dont le fœtus a pu se ressentir directement ou indirectement.

Pendant toute la durée de sa grossesse, la mère a travaillé dans une fabrique d'allumettes où son ouvrage consistait, du matin au soir, à ramasser de très lourdes charges de plaquettes de bois qu'elle portait, en les appuyant sur son ventre, aux ouvriers chargés de les tailler. Elle était (aux pièces») et obligée de se hàter continuellement.

Les charges de bois étaient «aussi lourdes qu'elle pouvait les porter)».

Qu'une telle besogne accomplie pendant toute la durée de la gestation ait pu entrainer un trouble dans le développement embryonnaire ou fœtal, on le conçoit facilement. Parmi les diverses explications qui se présentent à ce sujet, je considérerais comme la plus vraisemblable l'explication par une pression directe exercée à travers la paroi abdominale et utérine sur la tète du fœtus par un fardeau lourd et rigide, assez fréquemment porté par la mère pour qu'il dùt, à un moment ou à l'autre, atteindre l'enfant.

Il n'est pas nécessaire de supposer que la quantité de liquide amniotique ait été exceptionnellement faible, car on saitque, par la palpation, l'on peut souvent sentir, d'une façon très nette, les pieds, les genoux ou la tète du fœtus à travers la paroi abdominale, mème lorsque la quantité de liquide amniotique est normale.

On peut objecter que de très nombreuses femmes s'occupent à de très durs travaux pendant leurs grossesses sans donner le jour, pour cela, à des microcéphales. Mais il faut considérer qu'il s'agit ici de fardeaux rigides ramassés et portés à la hate, pendant des journées entières, durant toute la grossesse et toujours appuyés sur le ventre, ce qui est vraiment très rare. Une femme enceinte peut éviter et évite très généralement de por- ter ainsi des charges lourdes sur son abdomen; ou bien, si elle y est parfois obligée, comme il arrive parfois dans les campagnes, elle prend son temps et des précautions. Pour que la tète du fotus soit atteinte en pareil cas, il faut qu'elle se trouve justement exposée au choc à l'instant où il se produit; il faut une coïncidence vraiment peu probable. Tout autre est la condition d'une femme «travaillant à ses pièces» comme la mère de notre microcéphale, c'est-ì-dire devant accomplir en un temps donné le maximum de travail possible avec la régularité d'une machine, pliant son corps et le redres sant mille fois par jour pour faire ses paquets de bùches et les charger, puis, les transportant en toute hate, pour recommencer ensuite ind̦éfiniment, sans préjudice jour la besogne ordinaire de son ménage.

Pendant ses autres grossesses, le travail de cette pauvre femme a été tout différent. Elle travaillait soit seulement dans sa maison, soit à laver du linge au lavoir, travail pénible mais assurément moins périlleux pour le fœtus que le travail industriel ci-dessus décrit, - soit assise, dans une manufacture de tabacs.

Ainsi, sans pouvoir affirmer que j'ai saisi, dans le cas présent, la cause de la microcéphalie, je crois pouvoir dire que j'ai trouvé une cause paraissant très capable de produire cette monstruosité et l'ayant probablement produite. C'est cela qui, joint aux deux caractères pithécoïdes (canines et pli palmaire), relevés sur le microcéphale décrit ci-dessus, fait l'intérêt de ce cas particulier.
INSTITUT PALÉONTOLOGIE HUMAINE 